\title{
THE DISTRIBUTION OF NUCLEAR QUANTUM STATES IN "COLD" ROTATING NUCLEI
}

\author{
J. D. Garrett and J.R. German* \\ Physics Division, Oak Ridge National Laboratory, Oak Ridge, TN, 37831-6371 \\ L. Courtney \\ Department of Physics and Astronomy \\ University of Tennessee, Knoxville, TN 37996-1200 \\ CONF-9103176--3 \\ J.M. Espino \\ DE9 018254 \\ Universidad de Sevilla, Sevilla, Spain
}

\begin{abstract}
A statistical analysis of the distribution of level spacings for states with the same spin and parity is described in which the average spacing is calculated for the total ensemble. The resulting distribution of level spacings for states of deformed nuclei with $A=155$ 185 and $Z=62-77$ is the closest to that of a Poisson distribution yet obtained for nuclear levels. However, when only the even-spin, positive-parity data for even-even nuclei are considered, the level-spacing distribution becomes double peaked. The anomalously-large separations are shown to be the result of the low energy of the strongly-correlated, completely-paired yrast configuration of even-even nuclei. Average values of the level spacings also are discussed as a function of spin, parity, and nuclear type (even-even, even-Z-odd-N, etc.). Likewise, deviations from a Poisson distribution for several spacings (s) less than about $60 \mathrm{keV}$ are compared with similar values for $116 \mathrm{Sn}$ on an absolute scale. Such discrepancies are attributed to interactions (level repulsions) which become increasingly significant for $s \leq 60 \mathrm{keV}$.
\end{abstract}

\section{BACKGROUND INFORMATION}

It has become increasingly fashionable to consider the information content of the spectrum of eigenstates of a quantal system in terms of statistical concepts. ${ }^{1}$ The rich spectrum of nuclear states provides an excellent cpportunity for such analyses. Indeed, an analysis ${ }^{2}$ of the extensive, "complete," spectroscopic information near the neutron and proton thresholds (typically at an excitation energy of about $8 \mathrm{MeV}$ ) from neutron- and proton-resonance measurements ${ }^{3}$ for a variety of nuclei, shown in Figure 1, indicates that the distribution of the spacing of these levels with the same angular momentum, $I$, and parity, $\pi$, can be reproduced by a parameter-free distribution derivable from the predictions of the Gaussian orthogonal ensemble (GOE) of random-matrix theory. Of course, even though the distribution of level spacings is "given" by statistics, the average level spacing, $d(=1 / \rho$, where $\rho$ is the average level density), remains sensitive to the details of nuclear structure. Such level-spacing distributions have been taken as evidence 1.2 that the nuclear system at low spin and high excitation energy is "chaotic."

*Participant in the Oak Ridge Science and Engineering Research Semester from Winona State University, Winona, MN.

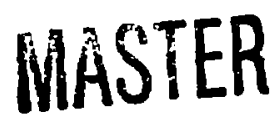

DISTRIBDTION OF FAIS DOCIMENT IS UNLIMITED 


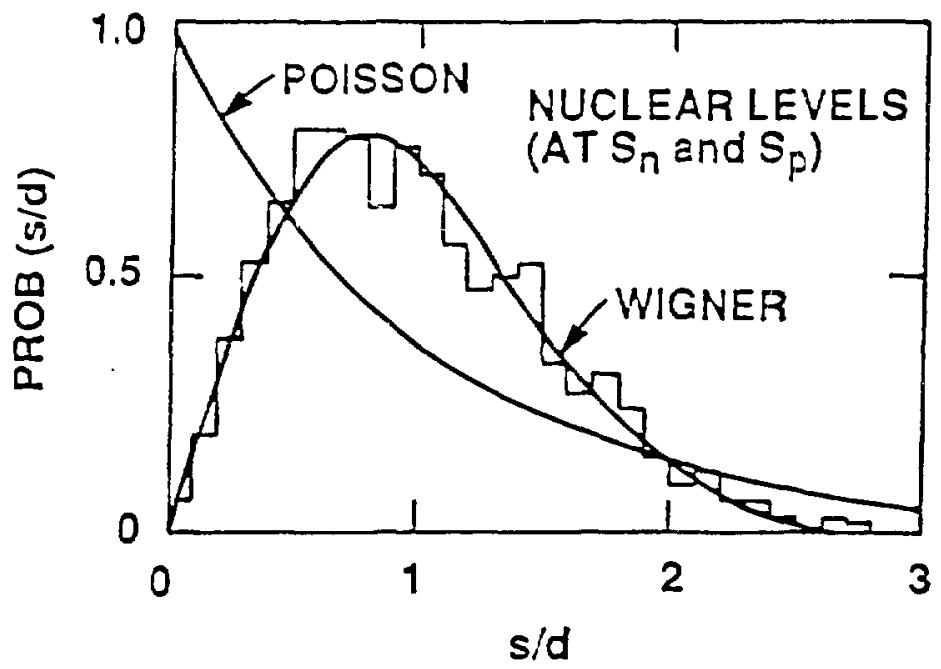

Fig. 1. Distribution of level spacings (s) normalized to the average spacing (d) for neighboring nuclear levels with the same spin and parity just above tne neutron- and proton-emission tisresholds. For comparison the Wigner (or GOE) distribution, a parameter-free distribution derivable from random matrices, and a Poisson distribution, assuming no correlation between levels with the same spin and parity, also are shown. This figure is taken from ref. 2 .

More recent analyses extend to lower excitation energies ${ }^{4-6}$ and attempt to extract information as a function of nuclear specie, ${ }^{4}$ for example, even-even versus odd-A, heavy versus light mass, or deformed versus spherical. Such analyses provide distributions intermediate to that expected for purely "ordered" (Poisson distribution) or purely "chaotic" (GOE or Wigner distribution) systems, see e.g. Figure 2, and a trend from GOE to Poisson proceeding from spherical regions to deformed regions, see refs. 4,6 . To obtain sufficient data for such analyses it is necessary to extend the analysis over a large range of energies and angular momenta. Since the level density increases exponentially with increasing excitation energy, the sensitivity to the lowest states is rapidly lost in such an extended analysis.

\section{ANALYSIS}

The present contribution reports a statistical analysis of the distribution of level spacings for states of deformed nuclei with $A=155-185$ and $Z=62-77$ hiving the same spin and parity. In contrast to earlier analyses, $2,4-6$ the average spacing is calculated from the complete data ensemble, instead of for each individual spin-parity group in a specific nucleus. This modified prescription is party justified by the fact that all levels considered are from deformed rare-earth nuclei, many of whose properties are known to be similar. For example, the moments of inertia calculated from the energy of the $22^{+} \rightarrow 20^{+}$yrastsequence transition of even-even nuclei in the $A=155-185$ mass region are equal within a five percent standard deviation, ${ }^{7}$ see Figure 3 . Such a criterion circumvents the usual problem of obtaining sufficient data to establish an accurate average level spacing, $d$, for each spin-parity group in a specific nucleus. For example, in the previous attempts to 


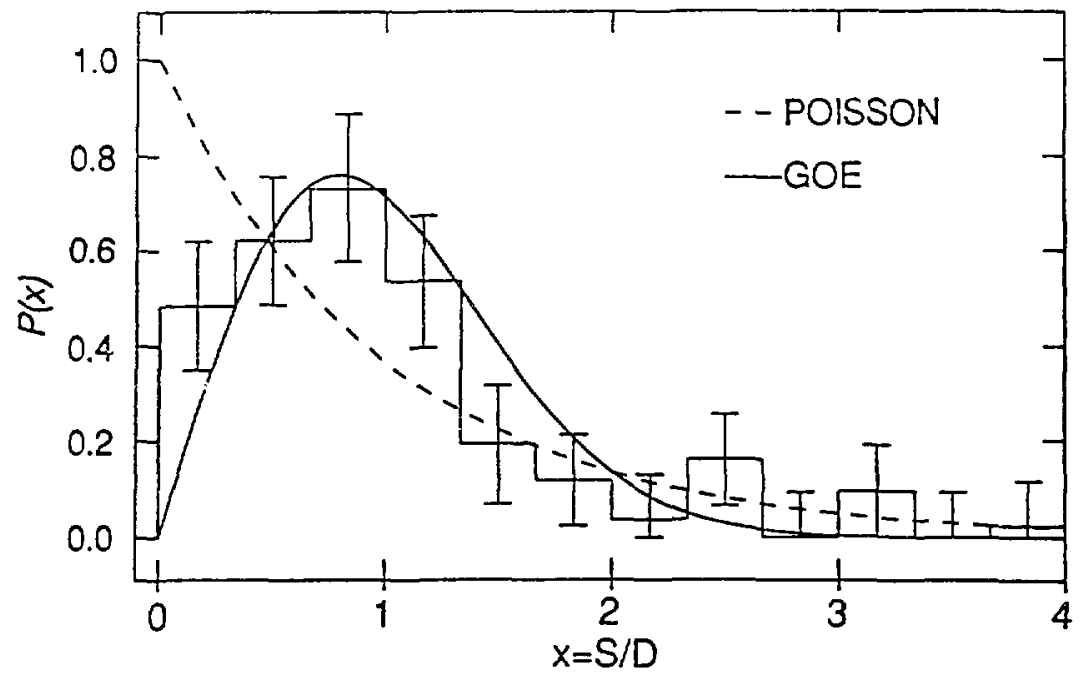

Fig. 2. Level-spacing distribution, taken from ref. 6 , for eighty-three $0^{+}, 1^{+}, 2^{+}, 3^{+}, 4^{+}$, and $5^{-}$states below $E_{x}=4.3 \mathrm{MeV}$ in ${ }^{116} \mathrm{Sn}$. See also the caption to Fig. 1 .

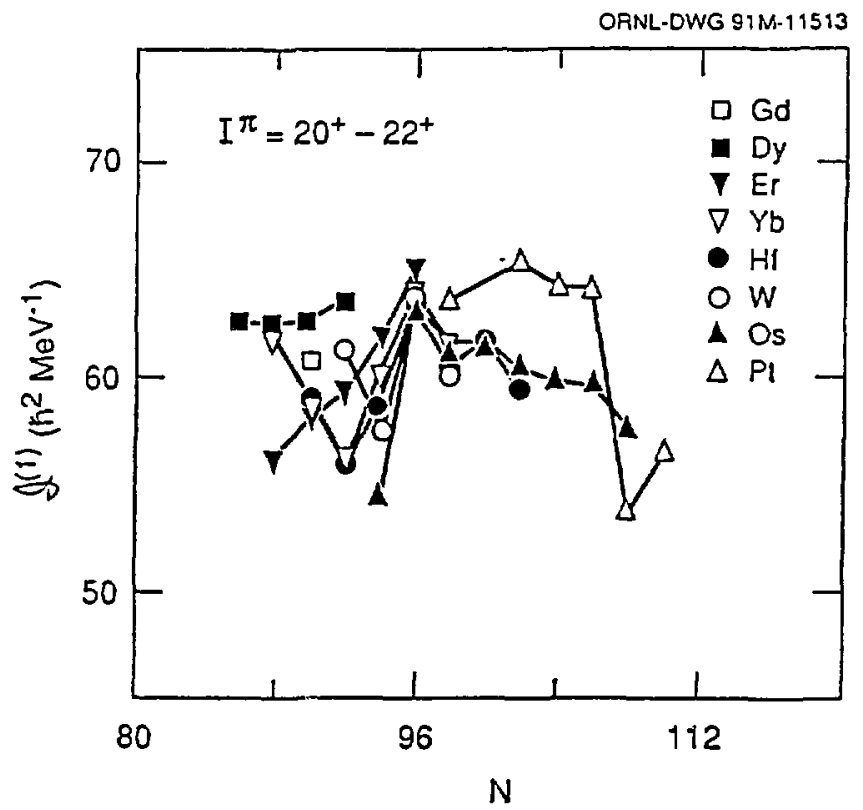

Fig. 3. Kinetic moments of inertia, $\ell(1)$, calculated for the $22^{+} \rightarrow 20^{+}$transitions of the yrast decay sequence of even-eyen rare-earth nuclei, are shown as a function of the neutron number. The values of $\ell(1)$ (average value $=60.6 \pm 3.0 \mathrm{MeV}^{-1} \hbar^{2}$ ) are surprisingly constant and do not show the $A^{5 / 3}$ dependence expected for a macroscopic rotor. These data are taken from and discussed in ref. 7. 


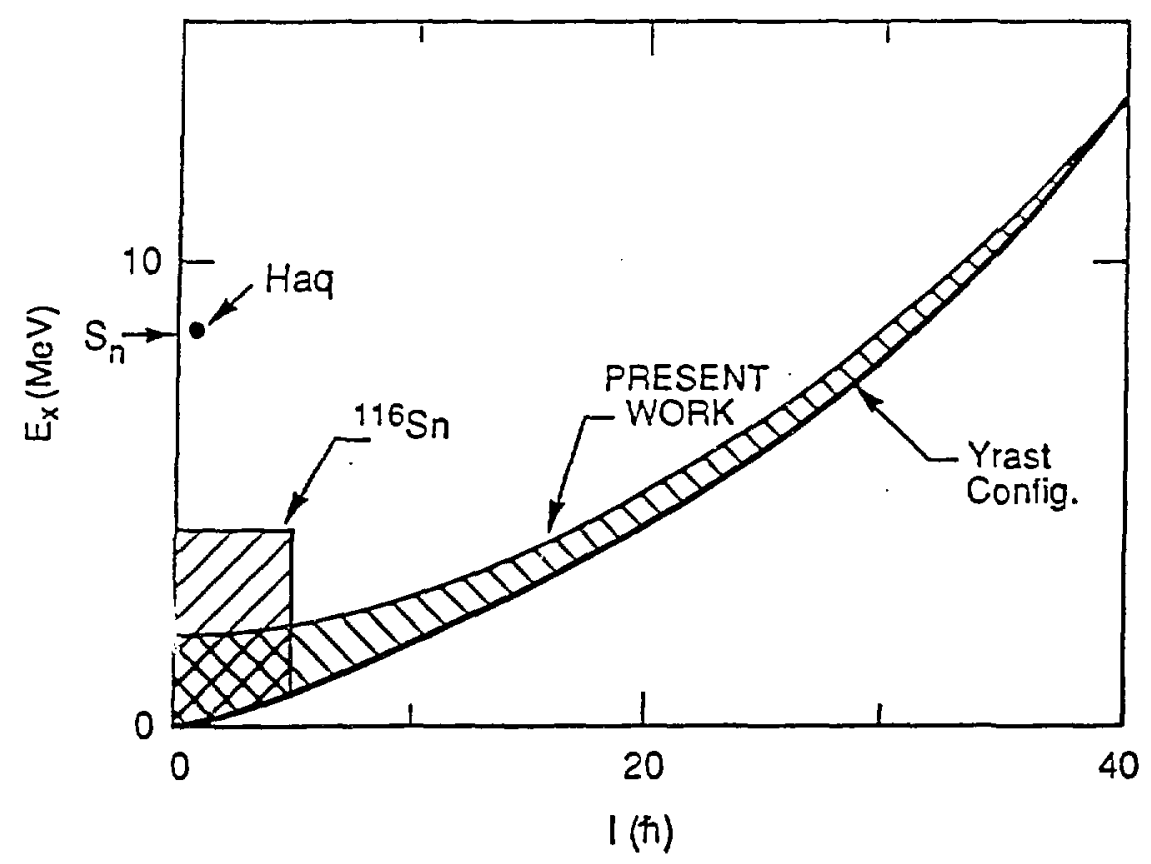

Fig. 4. Distribution as a function of excitation energy and spin corresponding to the data sets for which level-spacing distributions are shown in this work. Distributions for Hag, et al., ${ }^{116} \mathrm{Sn}_{3}{ }^{6}$ and the present analysis of $\mathrm{A}=155-185$ and $\mathrm{Z}=62-77$ data are shown in Figs. 1, 2, and 5-9, respectively. The average level spacings for these three analyses are of the order of $10 \mathrm{eV}, 160 \mathrm{keV}$, and $300 \mathrm{keV}$, respectively.

obtain information for some of the best data sets at low excitation energy often there were as few as 3-4 levels per spin-parity group for each nucleus. ${ }^{4}$ The added statistics obtained by relaxing this requirement on the average spacing allows for the first time (see however ref. 8) an analysis of level spacings for near yrast configurations -- see e.g. Figure 4 . This procedure also will provide sufficient statistics to obtain similar information specific to angular momentum, parity, and nuclear type (i.e. even-even, odd-even, etc.) for this mass region.

Of course, the relaxation of a criterion that requires several level spacings for each spinparity group leads to other problems (it always is necessary to pay a price for advantages gained). For example, the missing level problem (replacing two smaller spacings with a larger spacing) may become more severe producing a bias toward larger spacings. Conversely, larger spacings, being more difficult to establish from experiment, $u$ ill occur less frequently in the data sets producing a bias toward smaller spacings.

The experimental data for the even-even and odd-mass nuclei used in the analysis were obtained from the Evaluated Nuclear Structure Data Files (ENSDF) 9 available in November 1990 and from the Oak Ridge -- University of Tennessee High Spin Data Base. ${ }^{10}$ Level spacings for odd-odd nuclei were not included in the analysis, because such data sets are less complete and have fewer firmly established spin and parity assignments. Complete references to the 132 nuclei for which data have been included in the analysis is omitted from this preliminary report of our work. They can be obtained from the data bases.9.10 Data for $155-156 \mathrm{Sm}, 155,157,159 \mathrm{Eu},{ }^{155-161} \mathrm{Gd}, 155,157,159,161,163 \mathrm{~Tb}, 155-165 \mathrm{Dy}$, 


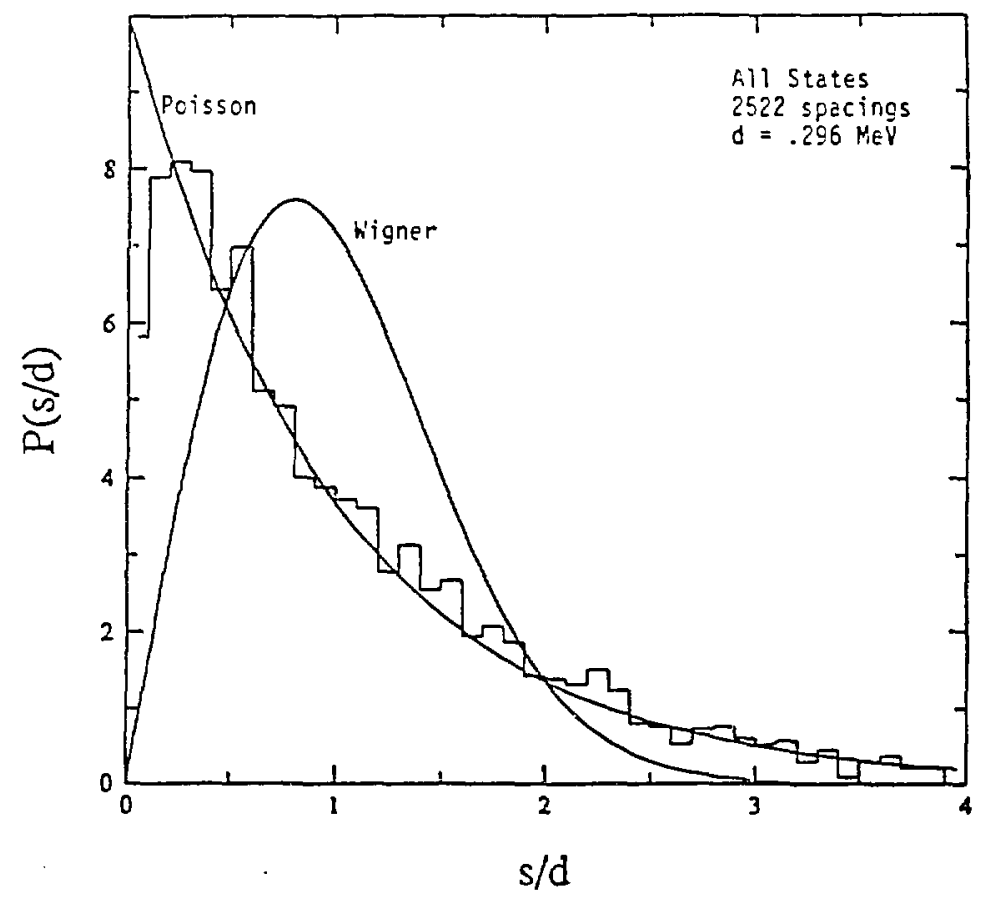

Fig. 5. Level-spacing distributions (histograms) for nuclear states with the same spin and parity for "cold" deformed rare-earth nuclei having $A=155-185$ and $Z=62-77$. For comparison, curves, corresponding to Poisson and Wigner distributions (often associated with "ordered" and "chaotic" behavior, respectively), are shown.

$157,159,161,163,165,167 \mathrm{Ho}, 156,158-171 \mathrm{Er}, 159,161,163,165,167,169,171 \mathrm{Tm}, 158,160-175$. $177-178 \mathrm{Yb}, 165,169,171,173,175,177 \mathrm{Lu}, 162,165-171,173-179 \mathrm{Hf}, 171,173,175,177,179 \mathrm{Ta}$, $170-172,175,178-185 \mathrm{~W}, 171,173,175,177,181,183,185 \mathrm{Re}, 177,179-185 \mathrm{O}$, and $179,181,183,185 \mathrm{Re}$ were used in the analyses.

\section{LEVEL-SPACING DISTRIBUTIONS}

The distribution of the 2522 level spacings included in the present analysis are compared in Figure 5 with calculated Poisson and Wigner distributions. (The Poisson and Wigner distributions usually are associated with "ordered" and "chaotic" systems, respectively. ${ }^{1}$ ) Except for the very smallest separations (histogram bins corresponding to the two smallest values of s/d shown in Figure 5), the agreement of the experimental spacings with the Poisson distribution is exceptional. Indeed these data are the closest to a Poisson distribution yet obtained for nuclear levels. Such an excellent Poisson distribution for the experimental level spacings is a surprise, since the missing level problem (raplacing two small spacings with a single large spacing) might be thought to be serious in this data set, which often contains only a single spacing for a particular spin-parity-nucleus group. (Indeed the deviations noted for the lowest spacings may be the result of missed levels; 

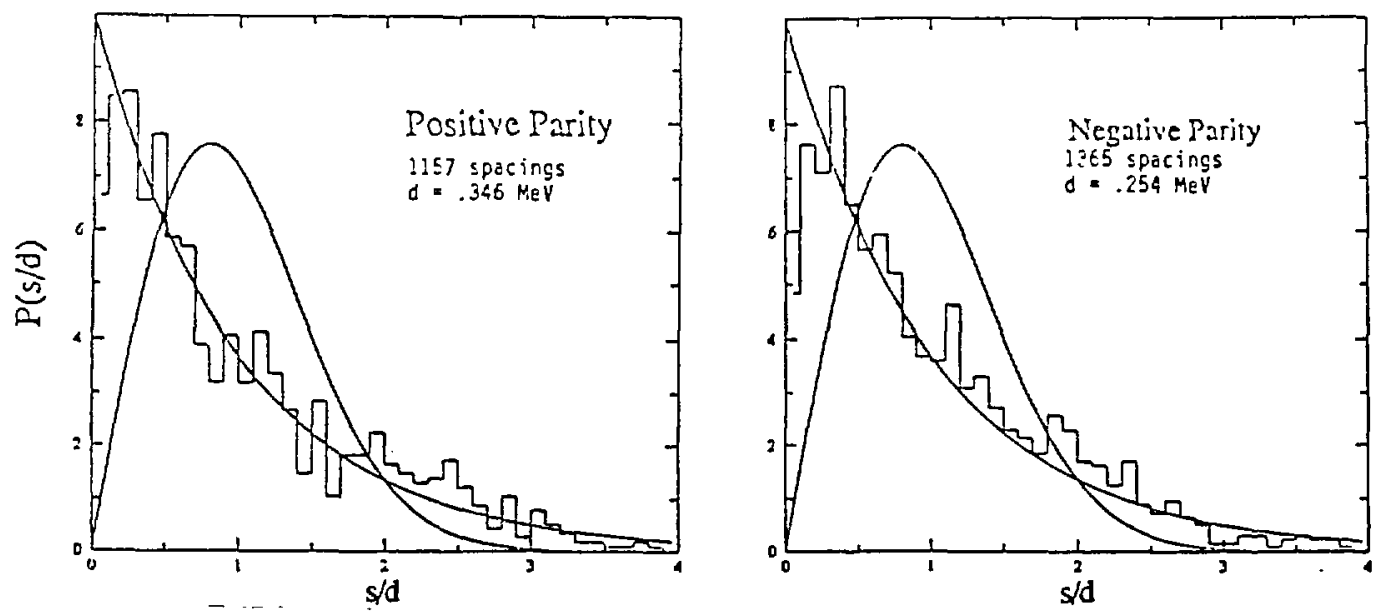

Fig. 6. Comparison of the level-spacing distributions for rare-earth states as a function of parity. See also the caption to Figure 5.
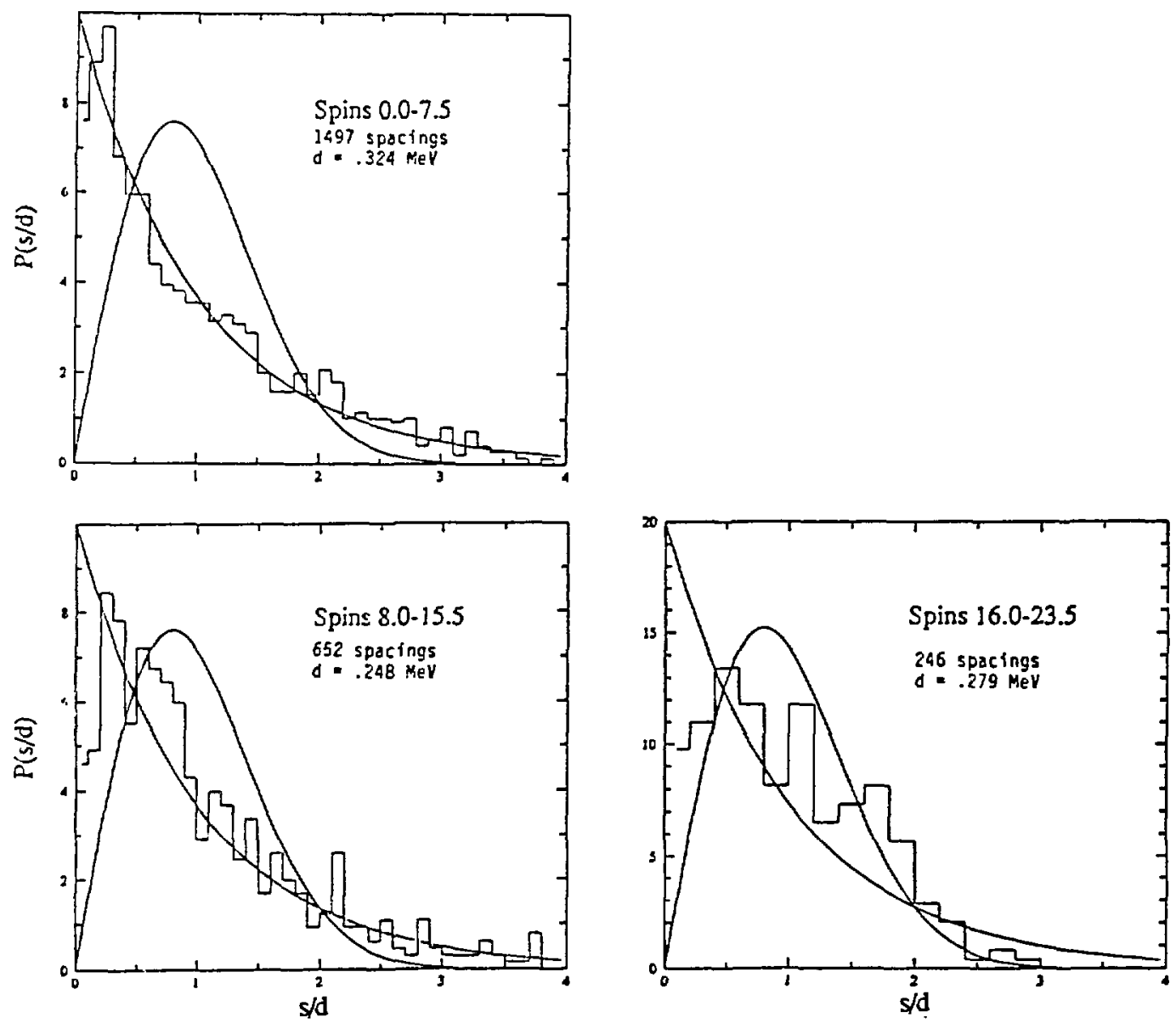

Fig. 7. Comparison of the level-spacing distributions for rare-earh states as a function of angular momentum. See also the caption to Figure 5. 

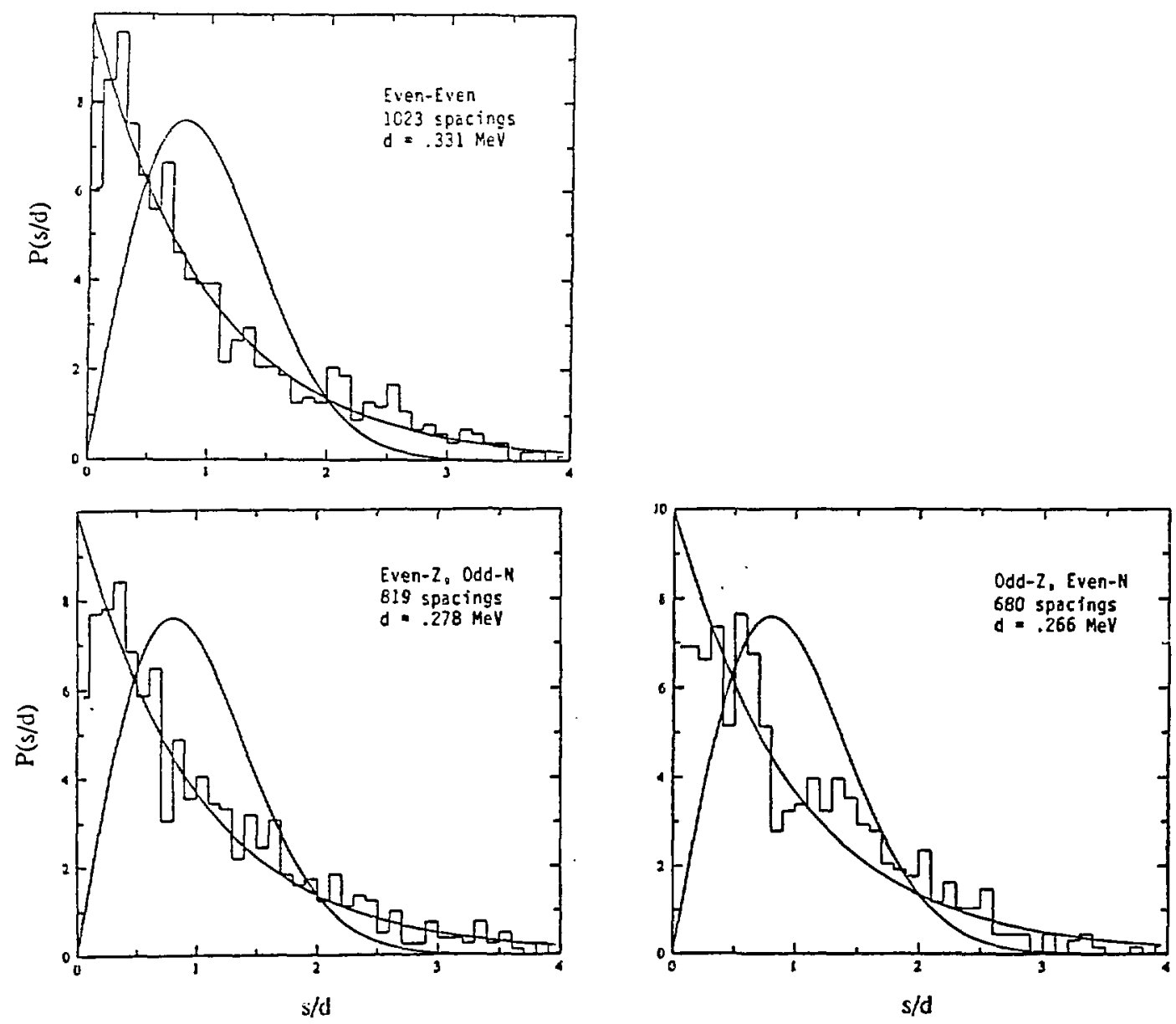

Fig. 8. Comparison of the level-spacing distributions for rare-earth states of eveneven, even-Z--odd-N, and odd-Z--even-N nuclei. See also the caption to Figure 5.

however, they also may indicate level repulsions for very closely-spaced levels, see the summary section.) The close agreement between these data and the Poisson distribution may be taken a posteriori as a partial justification of the assumptions of the anaiysis (see the preceding section).

The present analysis includes enough level spacings to allow some exclusive information to be obtained as a function of parity, angular momentum, and nuclear type (i.e. even-even, even-Z--odd-N, or odd-Z--even-N). Such analyses are shown in Figures 6-8. The experimental level spacings corresponding to positive- and negative-parity states (Figure 6), low- and intermediate-spin states (Figure 7), and even-even and even-Z-odd- $N$ nuclei (Figure 8) also have distributions close to the Poisson distribution. Of course, the statistical fluctuations in the distributions increase with the decreasing number of spacings in the sample, as expected. The distribution of the 680 spacings from odd-Z--even-N nuclei (Figure 8) deviates significantly from Poisson; neither is it Wigner. Even larger deviations from a Poisson shape is observed for the distribution corresponding to $16 \leq \mathrm{I}<$ 24. This distribution is intermediate between Poisson and Wigner. Likewise, the lack of very small spacings, observed in the analysis of all levels, persists for all the distributions shown in Figures 6-8. 

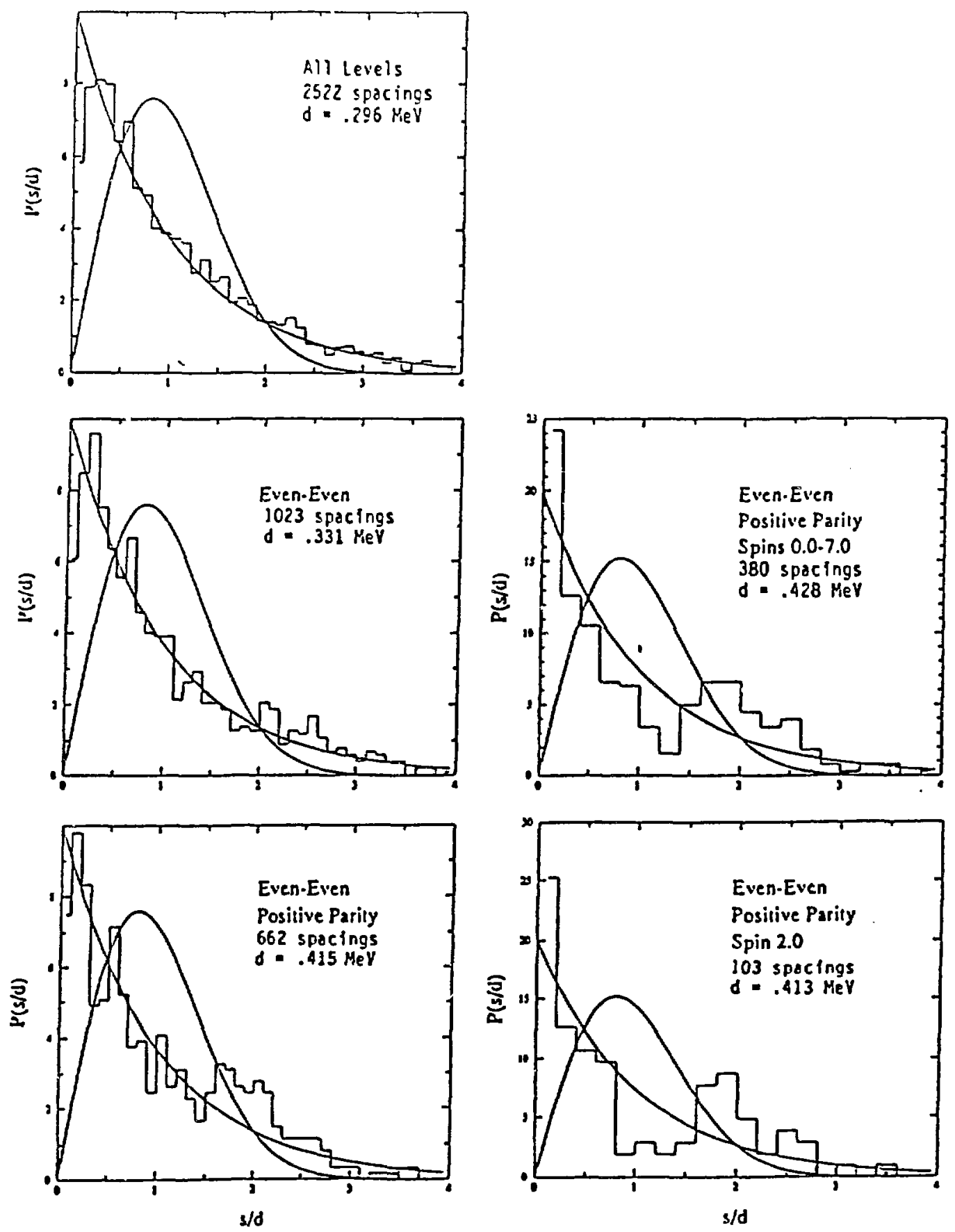

Fig. 9. Comparison of the level-spacing distributions of rare-earth states for a sequence proceeding from the complete data set to increasingly more exclusive conditions and ending with the $\mathrm{I}^{\pi}=2^{+}$states of even-even nuclei. The progression from a Poisson distribution for the complete data set to a double-peaked distribution for the $2^{+}$states is discussed in the text. See also the caption to Figure 5. 
A sequence of five level-spacing distributions based on various criteria, proceeding from the complete data set to increasingly more exciusive conditions and ending with 103 spacings for $\mathrm{I}^{\pi}=2^{+}$states in even-even nuclei, is shown in Figure 9. The strikingly Poisson-shaped distribution for the total ensemble becomes increasingly more complex as the conditions become more exclusive. Indeed, a peak appears in the data at s/d just less than 2 for the positive-parity states of even-even nuclei. The magnitude of this peak is enhanced when the data is restricted to low-spin, positive-parity levels in even-even nuclei. It becomes particularly pronounced when the level-spacing distribution for a single lowspin value of positive-parity states (e.g., the $2^{+}$states -- see Figure 9 ) in even-even nuclei is considered. Inspection of the data indicates that the spacings in the the $\mathrm{s} / \mathrm{d} \approx 2$ peak mostly correspond to that of the lowest pair of states (i.e., the spacing between the completely-paired, even-spin, positive-parity yrast states in even-even nuclei and the firstexcited states of the same spin in these nuclei). Of course, this spacing is not characteristic of the spacing of single-particle states. Instead it includes contributions from the correlation energy associated with pair correlations. 11,12 Similar conclusions have been made from a preliminary analysis of $I=19-20.5$ near-yrast data. ${ }^{13}$ The average level spacing, $d$, also increases as the large spacing between the completely-paired and the firstexcited states of the same spin in even-even nuclei becomes a larger fraction of the data (see Figure 9).

\section{AVERAGE VALUES OF THE LEVEL SPACINGS}

Except for a few cases, such as the $2^{+}$states of even-even nuclei shown in Figure 9 , the statistics of the data set is insufficient for extracting complete level-spacing distributions for each spin, parity, and nuclear type. However, meaningful average values of the level spacings can be obtained as a function of these quantities for a much wider range of values. Average level spacings are shown in Figure 10, as a function of angular momentum, for positive- and negative-parity, near-yrast states of even-even, even-Z-odd-N, and odd-Z-even-N nuclei with $A=155-185$.

For even-Z--odd-N and odd-Z--even- $\mathrm{N}$ nuclei, the average level spacings mostly represent average differences of quasiparticle energies, $E_{V}$, for configurations based on different Nilsson configurations of a specific parity. (Multiple-quasiparticle excitations, which will be important at higher excitation energies, and quasiparticle-vibrational coupled states do not dominate these data, which concentrate, especially at high spin values, on near-yrast configurations.) The quasiparticle energy is given byll

$$
E_{v}=\sqrt{\Delta^{2}+\left(\varepsilon_{v}-\lambda\right)^{2}}
$$

where $\varepsilon_{v}, \Delta$, and $\lambda$ are the energy of the single-particle Nilsson state, the pair-gap parameter, and the Fermi level respectively. Therefore, the average spacings should reflect, but not equal, the average spacing of the near-yrast, single-particle states of a specific parity. Indeed, they provide a lower limit for this value in the limit of a vanishing pair gap. The precipitous drop in the average level spacing, as a function of angular momentum, for small values of angular momentum is a result of the absence of states with $I<\Omega=K$ in rotational bands. That is, $\mathrm{d}(I=1 / 2)$ reflects the average spacings between $\Omega=1 / 2$ configurations, $d(I=3 / 2)$ reflects the average spacing between $\Omega=1 / 2$ and $3 / 2$ configurations, etc. For even-Z-odd-N nuclei the average spacing of positive-parity levels is consistently larger that of negative- parity levels of the same angular momentum -- see Figure 10. In contrast, the opposite is observed for odd-Z--even-N, $d(\pi=-)>d(\pi=+)$. 


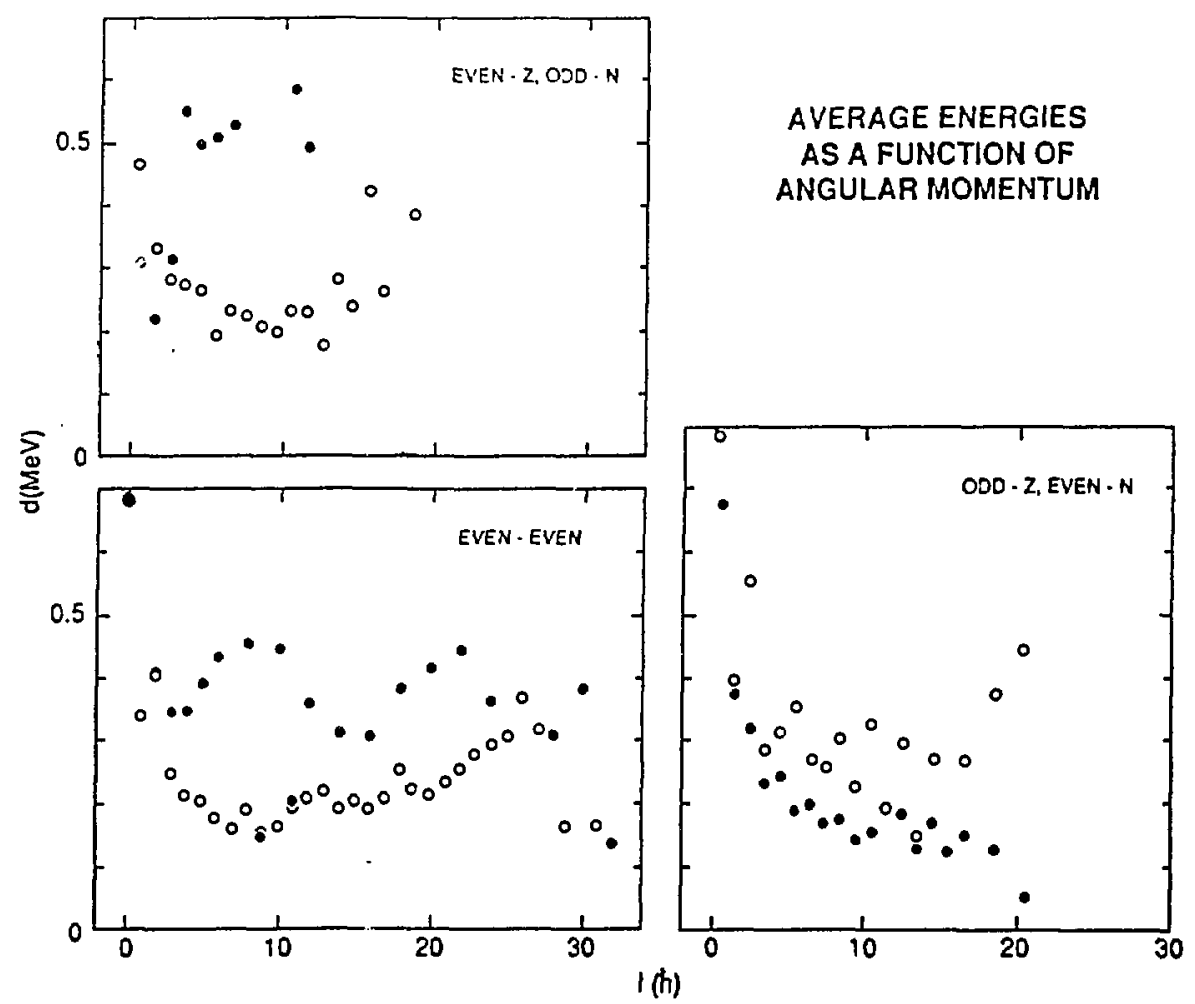

Fig. 10. Comparison of the distribution of average level spacings, $d$, as a function of angular momertum for the positive- and negative-parity levels (solid and open data points, respectively) of even-even, even-Z--odd-N, and odd-Z--even-N rare-earth nuclei.

These systematics agree with expectations. For the $A=155-185$ rare-earth nuclei the density of negative-parity, single-neutron states near the Fermi level is greater than that of positive-parity states. In the $N=82-126$ neutron shell there are 30 doubly-degenerate negative-parity neutron states (corresponding to the Nilsson components of the h9/2, $f_{7 / 2}$, $f_{5 / 2}, p_{3 / 2}$, and $p_{1 / 2}$ shell-model states) and 14 positive-parity states (corresponding to $i_{13 / 2}$ ) that span nearly the same range of excitation energies. For protons, which fill the next lower shell (with $20 \mathrm{~g} / / 2, d_{5 / 2}, d_{3 / 2}$, and $s_{1 / 2}$ positive-parity states and $12 h_{11 / 2}$ negative-parity states), the opposite is true. However, the reason that the overall average level spacings are less for the odd-Z--even- $N$ than for the ever $\mathrm{Z}$-odd- $\mathrm{N}$ nuclei (see Figure 10) remains unexplained. There are more single-neutron states than single-proton states in the valence shells; hence the spacing of single-neutron states would be expected to be less. Likewise, the slightly larger proton pair gap than neutron pair gap ${ }^{14}$ on average is not enough to explain this difference in rare earth nuclei where the proton and neutron oddeven mass differences are nearly equai.

For even-even nuclei the average level-spacing distributions are more complicated than for odd-A nuclei, due to the nixture of quasiparticle and strongly-correlated states (e.g., the completely-paired, even-spin yrast configuration and a variety of vibrational configurations) in the data set. In addition to the spacings of the various quasiparticle and correlated states, the relative number of each type of state in the data set also must be considered. For example, the spacing of $0^{+}$states is dominated by the large excitation energy difference between the ground-state and the bandhead of the beta band, whereas the 
spacing of $2^{+}$states also contains data for the smaller spacings between beta, gamma and two-quasiparticle bands. Hence, the average spacing decreases sharply between $0^{+}$and $2^{+}$. Likewise, the increase in $a^{2}$ erage spacings for $\mathrm{I}^{\pi}=4^{+}-8^{+}$is associated with the decrease of data for vibrational and two-quasiparticle states from $(n, \gamma)$ and transfer studies. Therefore, with increasing spin the data become increasingly dominated by (heavy-ion, $x n$ ) data, which for intermediate angular momentum are sensitive to the large spacing between the strongly-correlated, paired yrast configuration and that of the lowest-excited twoquasiparticle configuration ( $S$ band), based on the excitation of the most-alignable (i.e., those most strongly affected by the Coriolis force) quasiparticles. 15,16 Thus for $1^{\pi}=10^{+}$. $14^{+}$the spacing between the ground-state and $S$ bands decreases undil these bands cross at $I^{\pi}=14^{+}-16^{+}$, producing a minimum in the average level spacing distribution. Above the band crossing, of course, the spacings will increase.

Though octupole correlations may be important for negative-parity states, ${ }^{17}$ such correlations are expected to be less important in the average level spacing for the negativeparity states of even-even nuclei than the stronger, more-prevalent correlations of positiveparity states described in the preceding paragraph. Thus the level spacings for negativeparity states of even-even nuclei should reflect the spacing of the quasiparticle states to a greater degree than the positive-parity states. Indeed the level-spacing distribution for the negative-parity states of even-even nuclei follows a pattern similar to that observed for oddA nuclei.

\section{DISCUSSION AND SUMMARY}

A statistical analysis of level spacings is described in which the average spacing is calculated for the total data set, which is restricted to nuclei with similar properties. The resulting distribution of level spacings for nuclear states of deformed nuclei with $A=155$ 185 and $\mathrm{Z}=62-77$ is the closest to a Poisson distribution yet obtained for nuclear levels. In contrast to previous analyses, 2,4-6 these data are concentrated on near-yrast states with small intrinsic excitation energies, see Figure 4. Thus, the average level spacing is large; $\mathrm{d}=296 \mathrm{keV}$ for the total sample. Indeed this value is larger than the interaction matrix elements expected to mix these states. Therefore, neighboring states with the same quantum numbers are expected to retain individual character, i.e. to have distinctively different wave functions. This is the meaning of "order" in the present context. It, however, is tempting to ascribe the deviations from the Poisson distribution observed for the two lowest spacing bins shown in Figure 5 to the onset of level repulsion based on interactions that produce strong mixing between closely-spaced states. The lowest $(0-30$ $\mathrm{keV})$ and the next-lowest $(30-60 \mathrm{keV})$ bins contain only about 65 and $90 \%$ respectively of the probability of the Poisson distribution. Such deviations could be produced by interaction matrix elements $(\leq 30 \mathrm{keV})$ that perturb a significant fraction of the states for $s \leq$ $60 \mathrm{keV}$. A reduced probability for small spacings is observed in all the exclusive levelspacing distributions (see Figs. 6-8), indicating that reduced probability (relative to the Poisson distributior) for small spacings is spread systematically throughout the data. Missing levels also may contribute to such deviations, see the preceding discussion in the level spacing distribution section.

In contrast, previous analyses of the low excitation energy region ${ }^{4-6}$ with level spacing distributions intermediate between a Poisson and a Wigner distribution are based on data sets that extend to higher intrinsic excitation energies (i.e., the excitation energy relative to the yrast configuration) than that of the present analysis, see Figure 4. Thus many of the states in these data sets are more closely spaced than those of the present analysis. This is especially true, since the level density increases exponentially with increasing intrinsic 
excitation energy. For example, the average spacing of the twenty-eight $2^{+}$states in the $116 \mathrm{Cd}$ analysis, 6 which extends to an excitation energy of $4.3 \mathrm{MeV}$, is $111 \mathrm{keV}$, and a significant fraction of these spacings are less than $50 \mathrm{keV}$. Injeed, a comparison of the level spacing distribution of the present work and that of $116 \mathrm{Sn}$ on an absolute energy scale indicates that the maximum for the present analysis occurs at a larger spacing than that corresponding so ${ }^{116} \mathrm{Sn}$. However, this feanure is masked in the normalized distributions (compare Figures 2 and 5) by the vastly different average level spacing of these two data sets. At the neutron threshold, where the level-spacing distribution is that of a Wigner distribution, the average level spacing for $1 / 2+$ states established from s-wave capture on even-even targets are t.pically $1810 \mathrm{eV}$, a value much less than the interaction expected between these states.

Therefore, a coherent picture begins to emerge. When the nuclear states are well separated, such as the majurity of the states in the analysis described in the present work with $\mathrm{d} \approx 300 \mathrm{keV}$, each state contains detailed nuclear structure information, the levelspacing distribution is Poisson, and the situation is described as "ordered." In the other extreme, illustrated by the closely-spaced nuclear levels at ihe particle thresholds with $d=$ $10 \mathrm{eV}$, neighboring states have nearly-identical, strongly-mixed wave functions, the levelspacing distribution is Wigner, and the situation is described as "chaotic." That is, the wave functions of the individual states contain only average information on the ensemble of nuclear states in a specific excitation region. When $d \approx 100 \mathrm{keV}$, an intermediate situation emerges in which the level-spacing distribution is between that of a Poisson and a Wigner distribution. Indeed even the data from the present analysis show significant deviations from a Poisson distribution for $s<60 \mathrm{keV}$. In order to be quantitative with respect to the interaction strength at which the levels begin to mix producing the "transition from order to chaos," detailed analyses are needed for a wide range of excitation energies in the same mass region. At present the data is not adequate to completely characterize this "phase transition"; however, it is indicated that level repulsion becomes increasingly important for spacings less than about $60 \mathrm{keV}$.

The present analysis also illustrates how known correlations, such as the completelypaired state, can distort level-spacing distributions and average values of the level-spacing for exclusive data sets in which the spacing between such correlated and uncorrelated states is enhanced.

\section{ACKNOWLEDGMENTS}

The authors acknowledge informative discussions with S. Aberg, Y. Alhassid, C. Baktash, I.Y. Lee, B.R. Mottelson, S. Raman, J.F. Shriner, H.A. Weidenmuller, and J.Y. Zhang. Research was sponsored by the U. S. Department of Energy, under contract DE-AC05-840R21400 with Martin Marietta Energy Systems, Inc., and under contract DEFG05-87ER40361 with the University of Tennessee.

\section{REFERENCES}

1. O. Bohigas and H.A. Weidenmüller, Ann. Rev. Nucl. Part. Sci. 38, 421 (1988).

2. R. Haq, A. Pandey, and O. Bohigas, Phys. Rev. Lett. 48, 1986 (1982).

3. See e.g., G. Hacken, R. Werbin, and J. Rainwater, Phys. Rev. C 17, 43 (1978); E.G. Bilpuch, A.M. Lane, G.E. Michell, and J.D. Moses, Phys. Rev. C 28, 145 (1976); and refs. therein. 
4. T. von Egidy, A.N. Behkami, and H.H. Schmidt, Nucl. Phys. A454, 109 (1986); and Nucl. Phys. A481, 189 (1988); and J.F. Shriner, Jr., G.E. Mitchell, and T. von Egidy, Z. Phys. A338, 309 (1991).

5. G.E. Mitchell, E.G. Bilpuch, P.M. Endt, and J.F. Shriner, Jr, Phy's. Rev. Lett. 61, 1473 (1988); and Z. Phys. A335, 393 (1990).

6. S. Raman, T.A. Walkiewicz, S. Kahane, E.T. Jurney, J. Sa, Z. Gacsi, .L. Weil, K. Allaart, G. Bonsignori, and J.F. Shriner, Jr., Phys. Rev. C 43, 521 (1991).

7. J.M. Espino and J.D. Garrett, Nucl. Phys. A492, 205 (1989).

8. E. Costa, J. Flores, P. A. Mello, and E. Yepez, Phys. Letts. 53B, 32 (1974).

9. "Evaluated Nuclear Structure Data File edited and maintained by the National Nuclear Data Center, Brookhaven National Laboratory, on behalf of the International Network for Nuclear Structure Data Evaluation (March 1980). Summary of file contents and published documentation may be found on page iii of any issue of the Nuclear Data Sheets".

10. J.D. Garrett, M.A. Riley, C. Baktash, I.Y. Lee, N.R. Johnson, F. McGowan, L. Courtney, and L.L. Riedinger, in Oak Ridge National Laboratory Physic Division Progress Report (1988), ORNL-6508, pg. 70.

11. Aa. Bohr and B.R. Mottelson, Nuclear Structure (Benjamin, 1975, New York), Vol. 2, pgs. 647-53.

12. J.-Y. Zhang, J.D. Garrett, J.C. Bacelar, and S. Frauendorf, Nucl. Phys. A453, 104 (1986).

13. J.D. Garrett and J.M. Espino, in Proceedings of Conference on Nuclear Structure in the Nineties, ed. N.R. Johnson (ORNZ, 1990, Oak Ridge), vol. 1, p 126.

14. Aa. Bohr and B. R. Mottelson, Nuclear Structure (Benjamin, 1969, New York) Vol. 1, pgs. 169-171.

15. R. Bengtsson and S. Frauendorf, Nucl. Phys. A327, 139 (1979).

16. R. Bengtsson and J.D. Garrett, in Collective Phenomena in Atomic Nuclei, Int. Review. Nucl. Phys., vol. 2 (World Scientific, 1984, Singapore) pg. 193.

17. G. A. Leander, in Nuclear Structure 1985 ed. R. A. Bioglia, G. B. Herskind and B. Herskind (North Holland, Amsterdam, 1985) p. 249; and W. Nazarewicz, in Nuclear Structure 1985, ibid. p. 263.

18. S.F. Mughabghab and D.I. Garber, Neutron Cross Sections, BNL 325, Third ed. (National Technical Information Service, 1973, Springfield, Va.) vol. 1.

\title{
DISCLAIMER
}

\begin{abstract}
This report was prepared as an account of work sponsored by an agency of the United States Government. Neither the United States Government nor any agency thereof, nor any of their employees. makes any warranty, express or implied, or assumes any legal liability or responsibility for the accuracy. completeness, or usefulsess of any information, apparatus, product, or process disclosed, or represents that its use would not infringe privately owned ri ence herein to any specific commercial product, process, or service by trade name, trademark, manufacturer, or otherwise does not necessarily constitute or imply its endorsement, recommendation, or favoring by the United States Government or any agency thereof. The views and opinions of authors expressed herein do not necessarily state or reflect those of the United Sta'es Government or any agency thereof.
\end{abstract}

